\title{
Observation and simulation of wave breaking in the southern hemispheric stratosphere during VORCORE
}

\author{
M. Moustaoui ${ }^{1}$, H. Teitelbaum ${ }^{2}$, and A. Mahalov ${ }^{1}$ \\ ${ }^{1}$ School of Mathematical and Statistical Sciences, Arizona State University, Tempe, Arizona, USA \\ ${ }^{2}$ Laboratoire de Meteorologie Dynamique Ecole Normale Superieure, Paris, France \\ Correspondence to: M. Moustaoui (mohamed.moustaoui@asu.edu)
}

Received: 3 November 2012 - Revised: 18 February 2013 - Accepted: 19 February 2013 - Published: 16 April 2013

\begin{abstract}
An interesting occurrence of a Rossby wave breaking event observed during the VORCORE experiment is presented and explained. Twenty-seven balloons were launched inside the Antarctic polar vortex. Almost all of these balloons evolved in the stratosphere around $500 \mathrm{~K}$ within the vortex, except the one launched on 28 October 2005. In this case, the balloon was caught within a tongue of high potential vorticity (PV), and was ejected from the polar vortex. The evolution of this event is studied for the period between 19 and 25 November 2005. It is found that at the beginning of this period, the polar vortex experienced distortions due to the presence of Rossby waves. Then, these waves break and a tongue of high PV develops. On 25 November, the tongue became separated from the vortex and the balloon was ejected into the surf zone. Lagrangian simulations demonstrate that the air masses surrounding the balloon after its ejection were originating from the vortex edge. The wave breaking and the development of the tongue are confined within a region where a planetary Quasi-Stationary Wave 1 (QSW1) induces wind speeds with weaker values. The QSW1 causes asymmetry in the wind speed and the horizontal PV gradient along the edge of the polar vortex, resulting in a localized jet. Rossby waves with smaller scales propagating on top of this jet amplify as they enter the jet exit region and then break. The role of the QSW1 on the formation of the weak flow conditions that caused the non-linear wave breaking observed near the vortex edge is confirmed by three-dimensional numerical simulations using forcing with and without the contribution of the QSW1.
\end{abstract}

Keywords. Atmospheric composition and structure (Middle atmosphere - composition and chemistry)

\section{Introduction}

Wave breaking is a frequent phenomenon in the atmosphere, and represents one of the most important contributors to dynamics in the middle atmosphere. Wave breaking for Rossby waves involves irreversible deformation of a certain set of material contours, namely those of constant Potential Vorticity (PV), which would undulate reversibly under the influence of Rossby waves restoring mechanism (McIntyre and Palmer, 1983, 1984, 1985). In winter and spring, the stratospheric dynamics at high latitudes is dominated by the polar vortex. Rossby waves propagating upward near the vortex edge may reach large amplitudes at some levels, resulting in Rossby wave breaking (Haynes, 2005, and references therein). Under the effect of wave breaking, the stratospheric polar vortex experiences erosion that causes formation of steep gradients at its edge, and stirring of filaments into middle latitudes (Dritschel et al., 1991; Legras et al., 2001). These filaments are able to cause rapid transport of air from regions located inside the vortex to regions outside, and are important factors that regulate the distribution and exchange of ozone (and other gas traces) between the polar vortex and regions located in the surf zone (Plumb, 2002; Shepherd, 2007).

Breaking of Rossby waves in the stratosphere appears to be preferentially directed towards the equator, in that air pulled off the polar vortex edge tends to be transported towards middle latitudes (e.g. Polvani and Plumb, 1992; Waugh and Polvani, 2010). This was confirmed by a large number of observational and high-resolution modeling studies in the Northern Hemisphere (NH) (e.g. Waugh et al., 1994; Mariotti et al., 1997) as well as in the Southern Hemisphere (SH) (e.g. Teitelbaum et al., 1998). These studies 
have indicated that wave breaking is accompanied with development of filaments or tongues that irreversibly transport air from the vortex edge toward the equator. Other studies have also presented some events, although less common, where stratospheric wave breaking produced intrusion of mid-latitude air originating from the surf zone into the $\mathrm{NH}$ vortex core (Plumb et al., 1994; Mariotti et al., 1997). Moustaoui et al. (2003a) have reported events in the SH where anomalous and localized large ozone increases in the form of lamina were observed inside the ozone hole. They showed that these events were due to poleward transport into the core of the Antarctic vortex of air, with rich ozone within thin filaments originating from the vortex edge. The one-sidedness of wave breaking was attributed to cross-jet asymmetry of the vortex flow, which is such that Rossby waves break uniquely outward under most circumstances (Nakamura and Plumb, 1994).

Previous studies have shown that wave breaking in the troposphere and in the tropopause regions depends on the wave number (Orlanski, 2003; Riviere and Orlanski, 2007), and on the zonal variation of the zonal flow along jets (Peters and Waugh, 1996, 2003; Swanson et al., 1997; Swanson, 2000; Ndarana and Waugh, 2011; Esler and Haynes, 1999). It was suggested that wave breaking tends to occur in regions where the mean zonal wind is weak. Peters and Waugh (2003) examined the characteristics of Rossby wave propagation and breaking in the Southern Hemisphere upper troposphere during winter. They concluded that the location and the morphology of wave breaking will differ depending on the flow configurations. In the SH stratosphere, the polar vortex during winter is more symmetrical and centered near the pole compared to that in the NH (Randel and Newman, 1998; Waugh and Randel, 1999; Karpetchko et al., 2005). The wind along its edge presents little variations in the zonal direction. In spring, however, the SH stratospheric circulation is by far dominated by the quasi-stationary planetary wave with the zonal wavenumber 1 (QSW1) (Geller and Wu, 1987; Wirth, 1991; Bowman, 1993; Agosta and Canziani, 2011; Hartman, 1977; Randel, 1988; Moustaoui et al., 2003b). The QSW1 causes the vortex to be displaced from the pole or even split. The displacement of the vortex is accompanied by strong variation in the zonal wind (Moustaoui et al., 2003b).

One of the objectives of this paper is to analyze and explain a wave breaking event that occurred in the SH stratosphere in spring 2005, and which was registered by a balloon launched during VORCORE. Preliminary results for this event were presented for the first time in the SPARC meeting (Teitelbaum and Moustaoui, 2008). During VORCORE, several balloons were launched inside the Antarctic polar vortex. These balloons stayed and evolved inside the vortex core. This was expected, as the vortex edge presents a quasiimpermeable barrier to transport and exchange of air between regions inside and outside the polar vortex. There was, however, an exception for one balloon, which was also launched inside the polar vortex on 28 October 2005. This balloon was caught within a tongue of high PV produced by Rossby wave breaking and was ejected into the surf zone. This paper focuses primarily on this case. We analyze the flow conditions, the large and smaller scale dynamics, and the evolution of the polar vortex that were present during this event. Furthermore, we present high-resolution simulations using the method of contour advection with surgery (CAS) to demonstrate that air masses enclosing the balloon after its ejection were pulled off the vortex edge. The second objective of this paper is to study the role of the QSW1 and the asymmetry that this wave produces, in both the wind and the horizontal PV gradient, along the vortex edge on the location of the wave breaking event that was observed. The role of the QSW1 is confirmed with simulations using a three-dimensional isentropic model.

In Sect. 2, we present the data and the method used. In Sect. 3, we analyze the formation and the evolution of the tongue that caused the balloon ejection from the polar vortex. Results from high-resolution simulations of the tongue evolution, and the role of the flow configuration induced by the QSW1 on the formation of this tongue are presented in Sect. 4. Section 5 presents three-dimensional simulations of the polar vortex under real and idealized conditions, with and without the presence of the QSW1 forcing. Finally, conclusions are presented in Sect. 6.

\section{Data and method}

The data used in this study are taken from measurements made during the VORCORE experiment. This experiment took place in the Antarctic polar vortex for the period between September 2005 and February 2006. During this campaign of measurements, twenty-seven super-pressured balloons were launched inside the core of the vortex at altitudes located between 17 and $19 \mathrm{~km}$. All the balloons were instrumented to measure pressure and temperature. In addition, global positioning system (GPS) measurements were used to track the balloons locations with accuracies of $10 \mathrm{~m}$ in the horizontal and $20 \mathrm{~m}$ in the vertical. The data were transmitted to ground stations, through the ARGOS satellite system (Vincent et al., 2007). All the balloon locations and pathways that will be presented in this paper are the real isopycnic trajectories (along surfaces with constant density) observed by the balloon. The dynamics of the polar vortex will be investigated along isentropic surfaces because these surfaces are more relevant for adiabatic motion; which is a good assumption in the stratosphere for timescales of few days. The isentropic level that is considered in this study is $500 \mathrm{~K}$. This level corresponds roughly to an altitude that is around $19 \mathrm{~km}$, which is approximately the altitude where the balloon studied here evolved.

We use analyses from the European Centre of Medium Weather Forecast (ECMWF) for the period between 19 November and 25 November 2005 to study the wave breaking event observed during VORCORE. The 

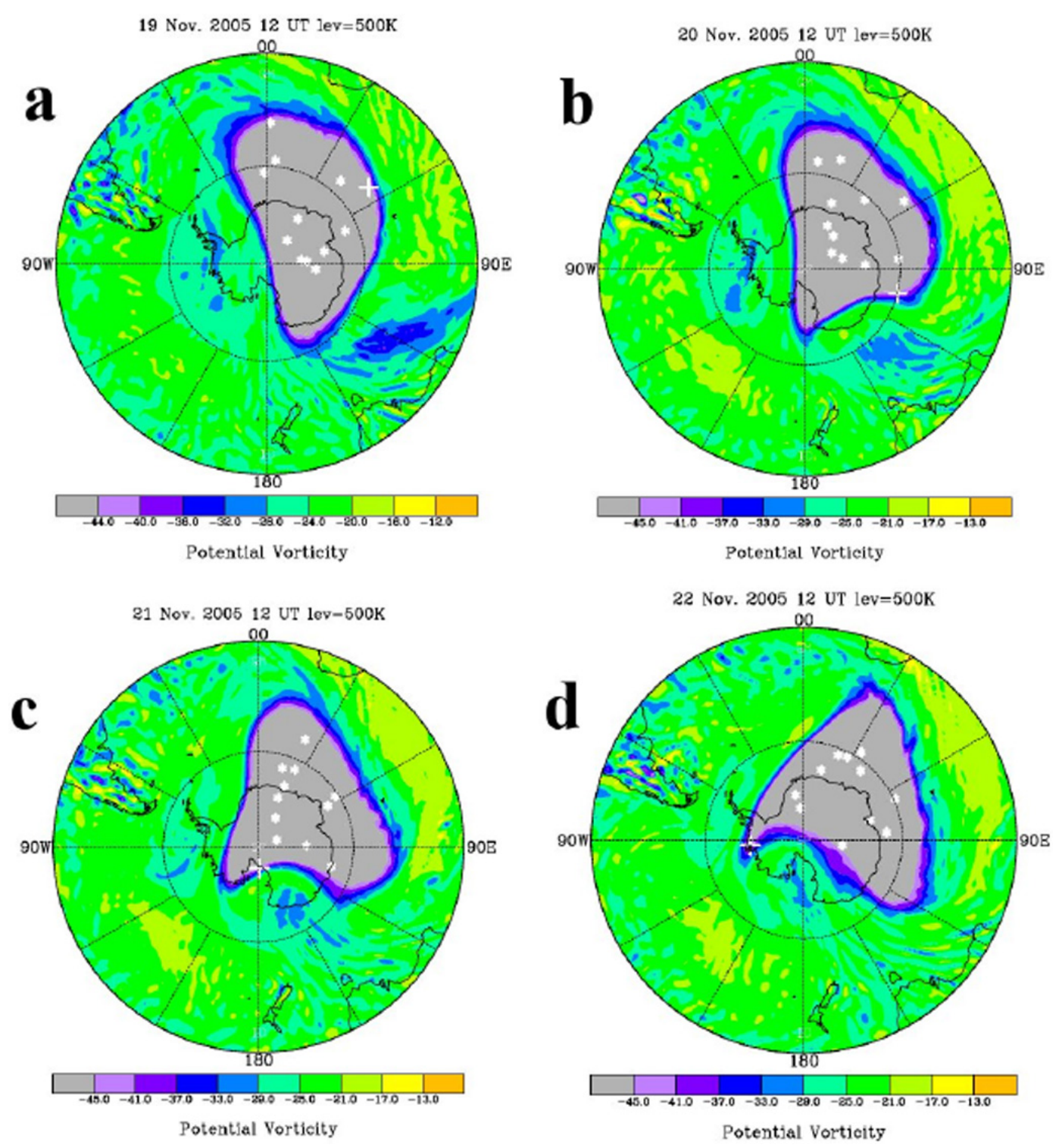

Fig. 1a-d. A sequence for the potential vorticity fields obtained from ECMWF at $500 \mathrm{~K}$ level on (a) 19, (b) 20, (c) 21, and (d) 22 November 2005 at 12:00 UTC. The contour interval is 4 PVU. The crosses superimposed in this figure indicate the locations of the balloon that was launched on 28 October 2005 and was ejected from the vortex. The stars denote locations of several balloons that evolved inside the vortex during the same period.

temperature, wind and geopotential fields from ECMWF are extracted on a grid with a horizontal resolution of 1 by 1 degree, and 21 pressure levels $(1000,925,850,700,500,400$, $300,250,200,150,100,70,50,30,20,10,7,5,3,2$ and $1 \mathrm{hPa}$ ). The PV fields are calculated from these data after interpolation on the $500 \mathrm{~K}$ isentropic level. The PV unit is PVU $=10^{-6} \mathrm{~m}^{2} \mathrm{~K} \mathrm{~kg}^{-1}$.

To compute the fine-scale structures of PV distributions, we use the CAS method (Mariotti et al., 1997; Waugh and Plumb, 1994; Moustaoui et al., 2003a). CAS is a Lagrangian method that reconstructs the evolution of a set of one or more contours of PV as it is transported by a flow. This method re- solves small-scale structures in PV; even when the flow fields used for advection have coarser spatial resolutions. CAS is based on the discretization of the PV field as a set of contours separated by finite PV jumps. Each of these contours is described by a number of nodes that are distributed along the contour. The surgery component of the CAS (Dritschel, 1989) consists of redistributing these nodes along the contour with density depending on the local curvature, splitting one contour in two or merging two contours of equal PV together when they are too close, and cutting out filaments that have become too thin to be significant. 

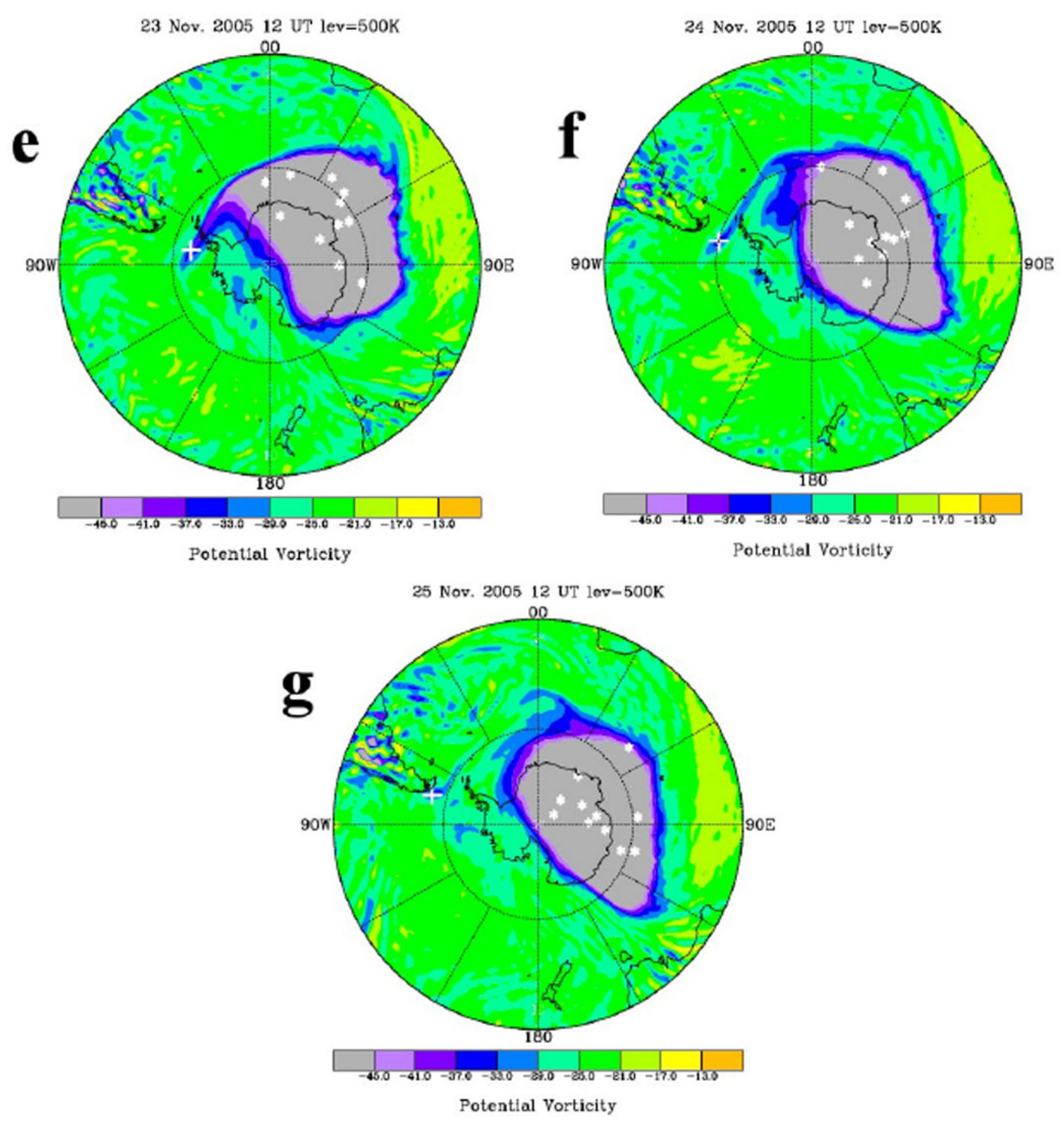

Fig. 1e-f. Same as Fig. 1a-d except that the potential vorticity fields are taken on (e) 23, (f) 24, and (g) 25 November 2005 at 12:00 UTC.

In addition, we use data from the National Center for Environmental Prediction (NCEP) Final Analysis (FNL from Global Forecasting System (GFS)) to initialize a threedimensional model that use potential temperature as a vertical coordinate. This model will be presented in Sect. 5. The FNL data are given every $6 \mathrm{~h}$ on standard pressure levels and a horizontal grid with a resolution of 1 by 1 degree. For the purpose of the model, these data are interpolated on 8 isentropic levels ranging from $350 \mathrm{~K}-700 \mathrm{~K}$.

\section{Observation of wave breaking and balloon ejection from the polar vortex}

In this section, we present the evolution of the Antarctic polar vortex for the period between 12:00 UTC 19 November 2005 and 12:00 UTC, 25 November 2005. Figure 1 shows a sequence of daily maps of PV distributions for the period between 19 and 25 November on 12:00 UTC from ECMWF at $500 \mathrm{~K}$. The symbols superimposed on Fig. 1 (the cross and the stars) denote the locations of several balloons launched during VORCORE. These balloons evolved in the SH stratosphere during the same period. The cross indicates the position of the balloon that was launched on 28 October 2005, and which will be ejected from the vortex at later times. The stars represent the locations of other balloons that were flying at the same time as the one represented by the cross, but stayed inside the vortex.

The distribution of PV on 19 November (Fig. 1a) shows that the polar vortex is disturbed by a large planetary wave, causing the vortex to be displaced from the pole (the presence of this wave will be shown in Fig. 2). In addition to 

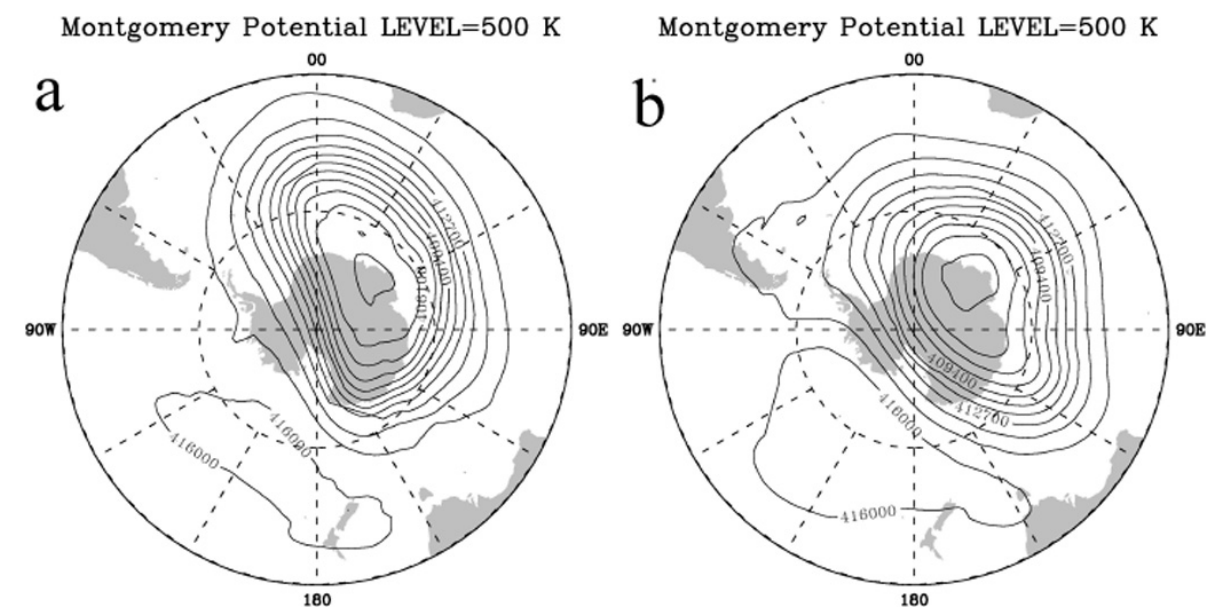

Fig. 2. Distributions of the Montgomery potential field at 500 K level from ECMWF: (a) on 12:00 UTC 19 November 2005, and (b) on 12:00 UTC, 24 November 2005. The unit is $\mathrm{m}^{2} \mathrm{~s}^{-2}$.

the planetary wave, the PV field shows a Rossby wave with a higher wave number propagating along the vortex edge. A closer look at the structure of the PV contours reveals that the presence of this wave causes a local distortion around the location $\left(60^{\circ} \mathrm{S}, 160^{\circ} \mathrm{E}\right)$, where Rossby wave breaking will be initiated (e.g. Fig. 1a-d). This wave amplifies as it propagates clockwise along the vortex edge, resulting in a local equatorward distortion of the vortex edge on 20 November. This distortion is now located at the longitude of $180^{\circ} \mathrm{E}$ (Fig. 1b). We note that on 19 and 20 November, all the balloons were evolving inside the polar vortex. On 21 November (Fig. 1c), the amplitude of the wave and the resulting distortion of the vortex edge become more pronounced. At this time, the balloon represented by the cross symbol is caught within this distortion. The amplitude of the wave continue to grow as the wave propagates leading to equator-ward wave breaking event on 23 and 24 November (Fig. 1e-f), and a development of a tongue of air masses with high PV values (in magnitude). On 25 November, this tongue is completely separated from the main vortex and appears as an isolated blob (Fig. 1g). The balloon, which was trapped within the tongue, is now pulled off the polar vortex and is located in the surf zone (the location of this balloon is represented by a cross in Fig. 1). The results presented in Fig. 1 show that the formation of the tongue and the ejection of the balloon from the vortex are due to a Rossby wave breaking event. We note that several other balloons, which were flying during the same times as the one that was ejected, were not caught within the wave breaking region, and stayed inside the polar vortex (the stars superimposed in Fig. 1 denote the locations of these balloons).

As noted above, the PV maps suggest the presence of a planetary wave and a smaller Rossby wave. The presence of the planetary wave is more evident in Fig. 2a. This figure shows the distribution of the Montgomery potential calculated from ECMWF analyses on 19 November at $500 \mathrm{~K}$. The Montgomery potential (or the dry static energy) represents, after scaling by the Coriolis parameter, the stream function for the geostrophic flow along isentropic surfaces (Andrews et al., 1987).

The distribution of the Montgomery potential (Fig. 2a) shows a clear evidence of presence of a Rossby wave signature with the wave-number 1 that is dominating the flow. Comparison between the gradients across the contours of the Montgomery potential indicates that the winds in the cyclonic part (e.g. $60-40^{\circ} \mathrm{S}$ and $0-90^{\circ} \mathrm{E}$ in Fig. 2a) of the wave are more pronounced than those found in the anticyclonic part (e.g. $60-40^{\circ} \mathrm{S}$ and $180-90^{\circ} \mathrm{W}$ in Fig. 2a). This is because the planetary wave prevents the vortex from being centered on the pole; and that the cyclonic winds associated with the displaced vortex add up to the cyclonic circulation induced by the wave. Figure $2 \mathrm{~b}$ shows the distribution of the Montgomery potential field on 24 November at $500 \mathrm{~K}$. This distribution is similar to the one found 5 days earlier (Fig. 2a), indicating that the observed wave is the QSW1. This wave has high amplitude in the stratosphere and dominates by far the flow in the Southern Hemisphere during spring (Agosta and Canziani, 2011; Hartman, 1977; Randel, 1988; Moustaoui et al., 2003b).

The presence of shorter propagating waves observed in the PV field is less clear in Fig. 2. These can be evidenced by plotting the vertical displacements of isentropic surfaces (Teitelbaum et al., 1998). Figure 3a presents the distribution of geopotential height on 19 November at $500 \mathrm{~K}$. This figure clearly indicates the presence of a Rossby wave with the longitudinal wave-number around 4 , and probably other shorter waves with smaller amplitudes. Figure $3 \mathrm{~b}-\mathrm{c}$ show the geopotential field at $500 \mathrm{~K}$ on days 22 and 24, respectively. Comparison between Fig. 3a-c indicates that these shorter waves are less stationary compared to the QSW1 (Fig. 2), with a relatively pronounced distortion of the altitude of the $500 \mathrm{~K}$ isentrope around the location $\left(60^{\circ} \mathrm{S}, 30-90^{\circ} \mathrm{W}\right)$ in Fig. 3c. This region coincides with the location where the tongue of 

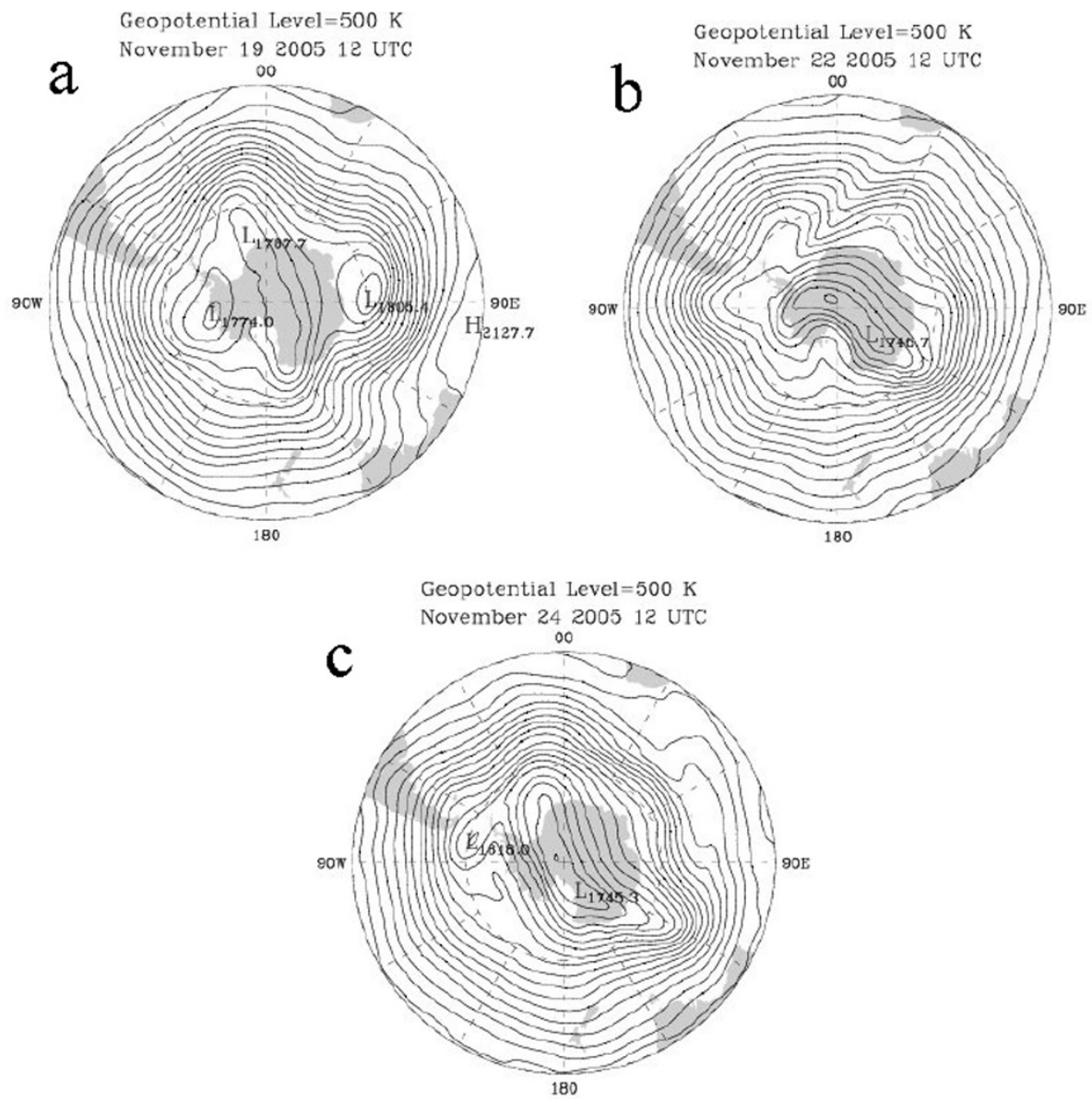

Fig. 3. Distributions of the geopotential field at 500 K level from ECMWF: (a) on 12:00 UTC, 19 November 2005, (b) on 12:00 UTC, 22 November 2005, and (c) on 12:00 UTC, 24 November 2005. The unit is $10 \mathrm{~m}$.

high PV was found (Fig. 1f), suggesting that these shorter waves are the ones that contribute to the observed Rossby wave breaking.

A regional zoom of the PV distribution on 24 November is presented in Fig. 4. We note the presence of small eddies that develop inside the tongue. According to McIntyre and Palmer (1984), such eddies could be produced by local instabilities because of the change in the sign of the gradient of PV across the tongue. Nevertheless, these eddies could also reflect the limitation of the ECMWF analyses to resolve the fine structures of these eddies as they became too narrow.

\section{Reconstruction of Rossby wave breaking and the role of the flow configuration induced by the QSW1}

\subsection{Reconstruction of Rossby wave breaking}

Before analyzing the role of the flow configuration induced by the QSW1, we will first show that the air masses enclosing the balloon after its ejection from the polar vortex are originating from the vortex edge. For this purpose, we re- constructed the PV fields using the CAS method. The initial PV distribution is derived from the ECMWF analyses on 12:00 UTC, 19 November 2005. The PV field used for initialization is discretized as a set of contours with different values. The magnitudes of the values of PV that are selected in the discretization are 28, 32, 36 and 40 PVU (absolute values). The contours of PV are then transported for 6 days starting from 12:00 UTC, 19 November 2005 up to 12:00 UTC, 25 November 2005. The wind fields used to advect the selected PV contours are obtained from analyses of the ECMWF winds, after interpolation of these fields to the $500 \mathrm{~K}$ isentropic level. Figure 5a-b show the fine-scale distributions of PV at $500 \mathrm{~K}$ reconstructed for 22 and 25 November using the CAS method. A thick filament or a tongue with high PV values is well reproduced and is found within the same regions as in the ECMWF analyses (Fig. 1d and g). In contrast to the fields found in the ECMWF analyses, the reconstructed PV distributions obtained from the CAS calculations show more details. In particular, the blob of high $\mathrm{PV}$ values enclosing the balloon that appears to be separated from the main vortex with respect to the analyses (Fig. 1g) is 


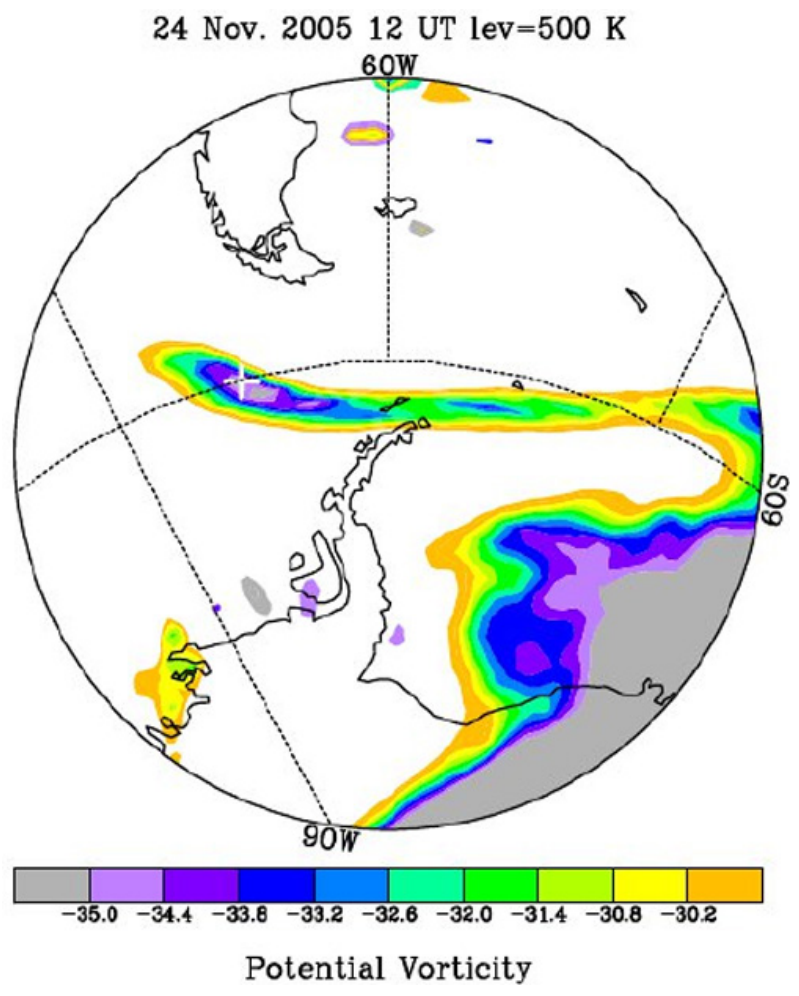

Fig. 4. A zoom for the isentropic potential vorticity distribution from ECMWF around the location of the tongue at $500 \mathrm{~K}$ on 12:00 UTC, 24 November 2005. The cross indicates the location of the balloon.

connected to the edge of the vortex through a thin filament in the CAS results (the balloon locations are indicated by dots in Fig. 5b). Thus, the reconstructed PV fields are able to resolve the small-scale filament connecting the blob of high IPV to the main vortex, and demonstrate that the air masses enclosing the balloon location after its ejection from the polar vortex are pulled from the vortex edge.

\subsection{The role of the flow configuration induced by the QSW1}

As stated in the introduction, many previous studies have shown that wave breaking is confined within regions of weak zonal winds (Nakamura and Plumb, 1994; Peters and Waugh, 1996, 2003; Swanson et al., 1997; Swanson, 2000; Waugh and Polvani, 2000). Moustaoui et al. (2003b) used similar arguments based on the climatology of the mean zonal flow to explain the existence of preferred regions for wave breaking and air exchange between high and low latitudes in the SH stratosphere during spring. Peters and Waugh (2003) identified four configurations for the mean zonal flow in the SH upper troposphere during winter including a single, broken and double jet. They showed that the location and the characteristics of wave breaking depend on the flow configuration.
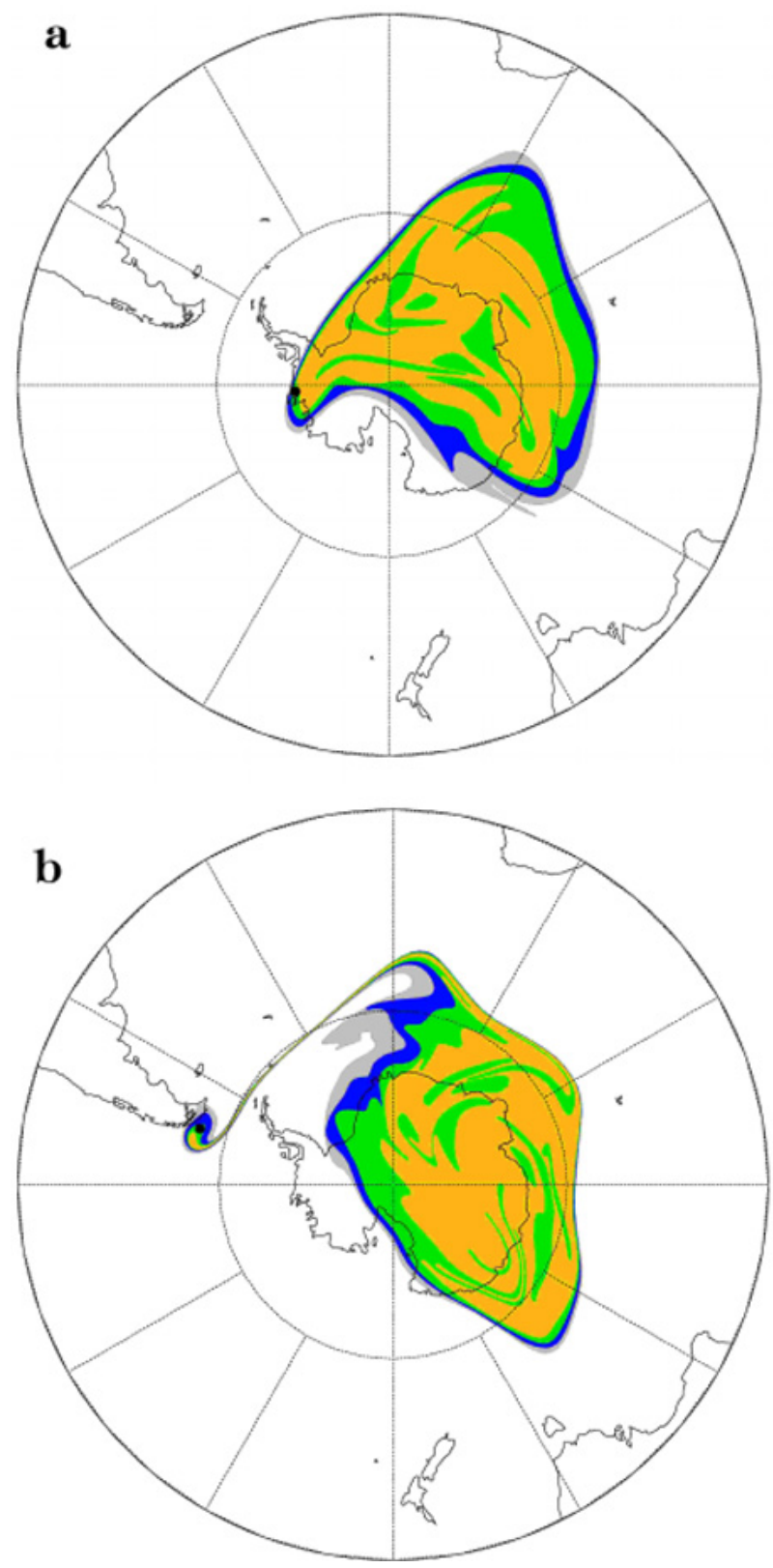

Fig. 5. Distributions of the isentropic potential vorticity field reconstructed by the CAS method at $500 \mathrm{~K}$ level: (a) on day 22 and (b) on day 25. The contours represented have a magnitude values of 28, 32, 36 and 40 PVU. The black dot indicates the location of the balloon.

Figure 6a shows the distribution of the background flow observed and averaged in time within 7 days between day 19 and day 25 November 2005. This period was chosen so that the contribution of the wave breaking event to the background flow is removed. An example of the instantaneous wind field observed on day 21 is shown in Fig. 6c. We consider the mean wind speed instead of the mean zonal flow (as opposed to previous studies) because the polar vortex in 


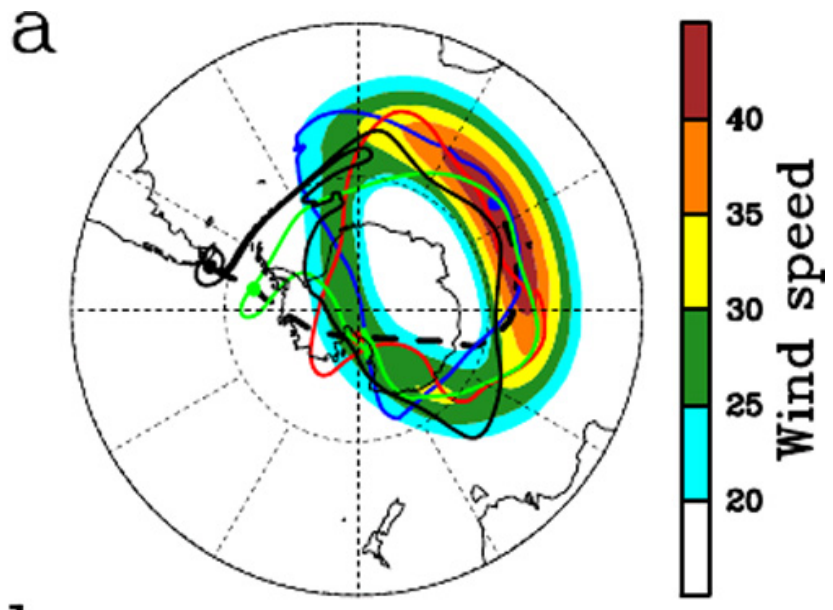

$\mathrm{b}$
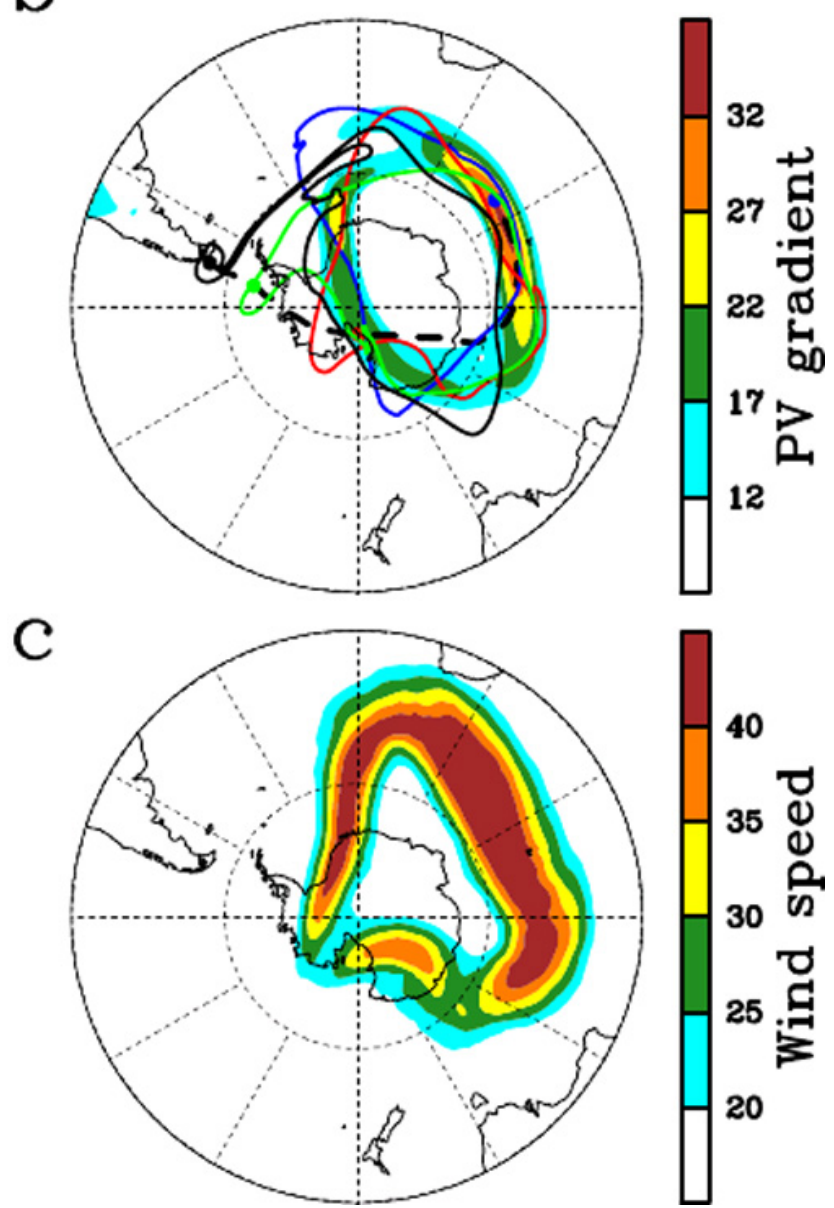

Fig. 6. Distributions of (a) the background wind speed $\left(\mathrm{m} \mathrm{s}^{-1}\right)$ and (b) the background horizontal PV gradient fields from ECMWF at $500 \mathrm{~K}$ level, averaged within the period between 19 and 25 November 2005. The black-dashed curve represents the real isopycnic trajectory of the balloon observed between day 19 and day 25. The colored curves represent the PV contour with the magnitude of $28 \mathrm{PVU}$ from the CAS calculations on day 19 (blue), day 21 (red), day 23 (green) and day 25 (black). For each day, the location of the balloon is denoted by a dot using the same color. (c) Distribution of the wind field obtained on day 21. the present case study was centered off the pole due to the presence of the QSW1. The background flow induced by the QSW1 shows asymmetry along the vortex edge, with a configuration that include a sector where the wind speed is weakening (lower-left part of the vortex edge) and a region where the QSW1 produces a localized jet (upper-right part of the edge). This structure is similar to the one referred to as a Single Jet (SJ) configuration of the upper tropospheric subtropical jet presented by Peters and Waugh (2003). We note that in our case, the localized jet is not related to the subtropical jet since the winds are taken in the stratosphere at the isentropic level of $500 \mathrm{~K}$, which is well above the location of the subtropical jet, and include both the zonal and the meridional components. The localized jet structure in our case is due to the presence of the QSW1, which tends to create asymmetry in the distribution of the wind magnitude along the edge of the polar vortex. The full isopycnic trajectory of the balloon observed between day 19 and day 25 is represented in Fig. 6a by the black-dashed line. We note that the balloon escapes the main polar vortex (the main polar vortex will be shown in Fig. 8a) in a region where the QSW1 induces weak winds. In Fig. 6a, we also superimposed the PV contour with the magnitude of $28 \mathrm{PVU}$ that was obtained from the CAS calculations on day 19 (blue), day 21 (red), day 23 (green) and day 25 (black). In each day, the balloon locations (represented by dots using the same color) were enclosed by these PV contours. These contours show the presence of Rossby waves with smaller wavelengths that propagate along the vortex edge. As these waves enter the sector where the winds are weak, they amplify and result in Rossby wave breaking. The wave breaking causes the balloon to be transported outside the main polar vortex on day 25 . Figure $6 \mathrm{~b}$ is the same as Fig. 6a except that here we plot the magnitude of the mean PV gradient, which is calculated as follows. First, The PV fields at $500 \mathrm{~K}$ are averaged within 7 days. The resulting mean PV field is used to calculate the magnitude of the total PV gradient (including both the eastward and the northward components). Then the mean PV gradient is filtered to remove the noise using a spectral filter. The QSW1 induces asymmetry in the mean PV gradient along the vortex edge. In the region where the PV gradient is high (upper right) the amplitude of Rossby waves is small because of the strong restoring force due to the high PV gradient. As these waves enter the region where the PV gradient is weak (lower left) they amplify and break. This is consistent with previous studies which have suggested that the PV gradient tends to inhibit wave breaking (Hitchman and Huesmann, 2007; Haynes et al., 2007).

\section{Simulations using a 3-D isentropic model}

In order to investigate the role of the QSW1 and the induced mean wind configuration on the polar vortex dynamics and wave breaking, we conducted simulations using 

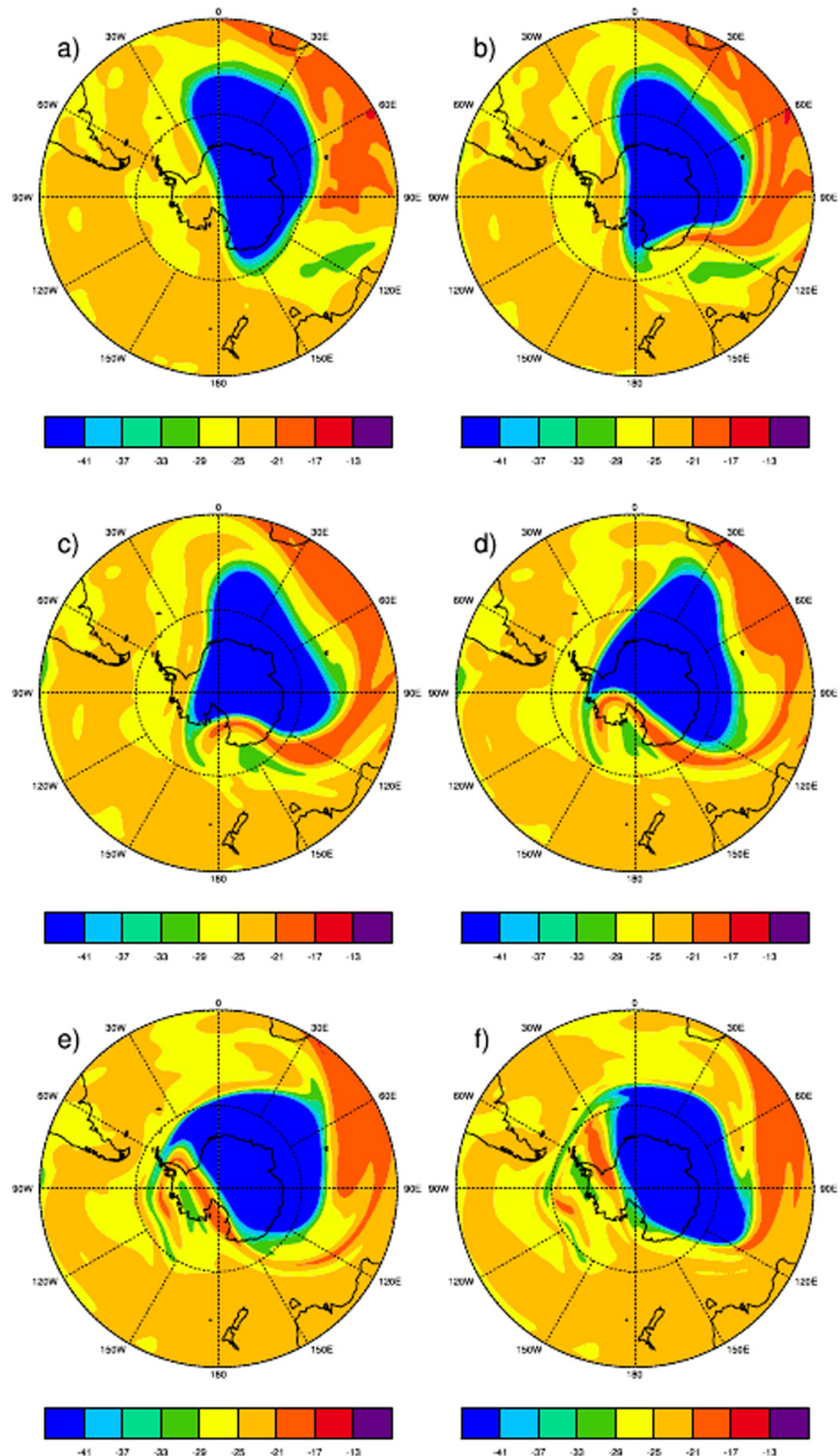

Fig. 7. A sequence for the magnitude of potential vorticity fields obtained from the 3-D isentropic model simulations at $500 \mathrm{~K}$ level on day (a) 19, (b) 20, (c) 21, (d) 22, (e) 23, and (f) 24 November 2005 at 12:00 UTC. 

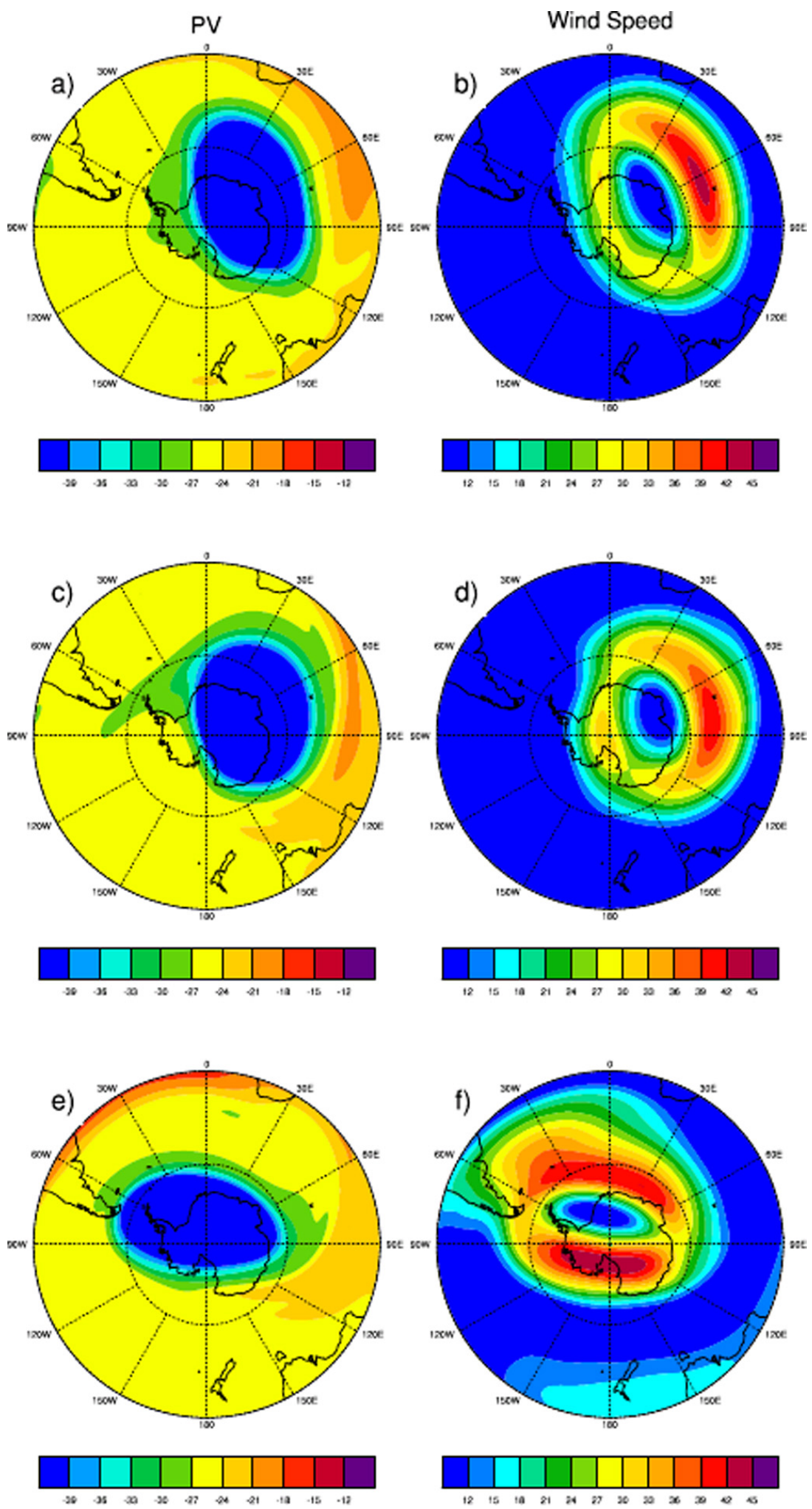

Fig. 8. Distributions of PV (a) and the mean wind speed (b) at $500 \mathrm{~K}$ used to initialize two idealized simulations. These fields are obtained from the NCEP-FNL analyses after averaging the instantaneous fields between 19 and 25 November 2005. (c) and (d) show the PV and the wind fields simulated after 5 days. (e) and (f) are the same as (c) and (d) except that the forcing associated with the QSW1 is removed from the boundary conditions used (the pressure at the top $(700 \mathrm{~K})$, and the geopotential at the bottom $(350 \mathrm{~K})$ ). 
a three-dimensional model that solves the primitive atmospheric equations under both real and idealized conditions. The model equations are written in the coordinate system in which the vertical coordinate is potential temperature. We are only considering adiabatic effects in the model. Under this assumption, the momentum, the continuity and the hydrostatic equations take the forms

$$
\begin{aligned}
\frac{d \boldsymbol{V}_{\mathrm{h}}}{d t}+\nabla M+f \boldsymbol{k} \times \boldsymbol{V}_{\mathrm{h}} & =\mathbf{0} \\
\frac{\partial \sigma}{\partial t}+\nabla\left(\sigma \boldsymbol{V}_{\mathrm{h}}\right) & =0 \\
\frac{\partial M}{\partial \theta} & =\Pi,
\end{aligned}
$$

where $\boldsymbol{V}_{\mathrm{h}}$ is the horizontal velocity vector along the isentropic coordinate, $f$ is the Coriolis parameter, $\theta$ is the potential temperature, $\sigma$ is the isentropic density given by

$\sigma=-\frac{1}{g} \frac{\partial p}{\partial \theta}$,

$p$ is the pressure, $\Pi=c_{\mathrm{p}}\left(p / p_{\mathrm{r}}\right)^{\kappa}$ is the Exner function, and $M$ is the Montgomery potential, which can be written in terms of the Exner function as $M=\theta \Pi+g z$.

The above equations are solved on the sphere using the divergence-vorticity form of the equations. The numerical model is spectral in the horizontal with a triangular spectral truncature of T106. In the vertical, 8 isentropic levels are used with staggered grid spacing where the vorticity, the divergence and the Montgomery potential are placed half a grid point away from the pressure and the Exner function. The vertical isentropic levels are uniformly distributed between $350 \mathrm{~K}$ and $700 \mathrm{~K}$, with a grid spacing of $50 \mathrm{~K}$. The numerical scheme uses a third order Runge-Kutta method. The simulations are driven by the FNL analyses. At the upper and the bottom boundaries, we impose the pressure (at $700 \mathrm{~K}$ ) and the geopotential (at $350 \mathrm{~K}$ ), respectively. The geopotential at the bottom boundary is used to specify the wave forcing.

\subsection{Realistic simulation}

In the first simulation, we conducted a 5-day real simulation of the polar vortex dynamics starting on 19 November at 12:00 UTC and ending on 24 November 2005 at 12:00 UTC. The initial conditions are interpolated from the analyses on day 19. The upper boundary conditions use a time-independent pressure distribution obtained from a temporal average of the NFL pressure fields at $700 \mathrm{~K}$ between 19 and 25 November. At the bottom boundary, we use the instantaneous geopotential distributions obtained by interpolating the geopotential fields from the FNL analyses at $350 \mathrm{~K}$ to the time step and to the grid point locations of the simulation. Figure 7 shows the daily evolution of the PV distribution at $500 \mathrm{~K}$ simulated by the model from 19 to 24 November. The remarkable agreement between the fields presented in this figure and those obtained from the ECMWF analyses (Fig. 1a-f) demonstrates that the model was able to predict the real evolution of the polar vortex, including development of the high IPV tongue, even though our model is relatively simple in that it uses a time-independent top pressure distribution; as opposed to the ECMWF analyses, there is no diabatic heating, neither parameterization nor assimilation that are included in our model. Comparisons between the simulated PV fields, which are presented in Fig. 7, and the distribution of the 6-day temporal mean wind field at $500 \mathrm{~K}$ (Fig. 6) shows that as Rossby waves enter the geographical sector where the QSRW1 induces weak winds, the waves amplify and result in breaking. Thus, this suggests that, in addition to causing the displacement of the polar vortex off the pole, the QSRW1 induces an asymmetry of the winds along the vortex edge, and creates a preferred region for wave breaking where the mean winds are weak. This explains the location where the balloon was ejected during the VORCORE experiment.

\subsection{Idealized simulation}

To confirm the role and the importance of the QSW1 on the modification of the mean winds and its consequence in the development of wave breaking, we conducted two idealized simulations. In one of these simulations, all the fields used in the model are obtained from the FNL analyses after averaging in time within 7 days (between day 19 and day 25) both the initial and the boundary conditions. In this case, the prescribed pressure at the top and the geopotential at the bottom boundaries are both averaged and are fixed (time independent). Figure $8 \mathrm{a}-\mathrm{b}$ shows the temporal mean of $\mathrm{PV}$ and the wind fields used for the initialization. This figure shows that the mean wind speed distribution is asymmetric along the vortex edge. The QSW1 induces higher winds along the upper right region of the vortex, and low winds in the lower left part of it. These fields, after 5 days' evolution, are shown in Fig. 8c-d. The distributions of the wind speed show that the QSW1 still dominates the flow (Fig. 8d). The PV field shows a formation of a tongue of high IPV values in the region where the winds are weak (Fig. 8c).

The second idealized simulation was conducted using a model setup that is similar to the previous one, except that the upper and lower boundary conditions are modified so that the QSW1 contribution is removed. Thus, there is no forcing associated with the QSW1 at the bottom boundary. We note however that in this case the initial conditions still include the contribution of the QSW1, and are similar to the one used in the previous case (Fig. 8a-b). Figure 8e-f show the PV and the wind fields simulated after 5 days. By suppressing the forcing due to the QSW1, the tongue of high PV found when this forcing was turned on does not develop (Fig. 8e). Comparison of Fig. 8c-d and Fig. 8e-f shows clear evidence that in the presence of the QSW1, the vortex remains centered off the pole, the asymmetry in the wind field induced by this 
wave along the vortex edge persists and the tongue of high PV develops. In contrast, when the forcing due to this wave is removed, the polar vortex tends to move and to be centered on the pole, the initial contribution of the QSW1 decays with time, and the tongue of high PV does not form. These two idealized simulations confirm the role of the QSW1 on the configuration of the wind speed along the polar vortex edge, and demonstrate the role of the QSW1 on the location of Rossby wave breaking that caused the observed ejection of the balloon from the polar vortex during the VORCORE experiment.

\section{Conclusions}

We studied lower stratospheric dynamics in the Antarctic region associated with Rossby wave breaking event that occurred in spring of 2005 and was registered by a balloon launched during the VORCORE experiment. In the VORCORE campaign of measurements, several superpressured balloons were launched inside the polar vortex. We showed that all these balloons evolved inside the vortex for several days, except one of them. In this case the balloon was caught in a wave breaking event, and was pulled off the vortex within a tongue of high PV. The formation and the evolution of this tongue were studied in details. We analyzed the evolution of the planetary waves that were present during the wave breaking event and the subsequent formation of the tongue. The structure of the potential of Montgomery showed the presence of a QSW1, which dominated the Rossby wave field and resulted in the global distortion of the polar vortex by preventing it from being centered on the pole. Other shorter waves propagating along the vortex edge causing local distortions of the vortex edge were also identified from the distribution of the PV maps and the geopotential height field.

The evolution of the tongue was examined and followed for the period starting on 19 November 2005 and ending on 25 November 2005. During the last three days of this period, the balloon was caught within a tongue of air masses with high PV originating from the polar vortex and was transported towards the Equator. The balloon was thereafter ejected from the main vortex.

High-resolution simulations using the CAS technique reproduced the evolution of the observed wave breaking event and the formation of the tongue. They confirmed that the air masses surrounding the balloon location after its ejection were stripped from the polar vortex. The formation and the location of the tongue were explained by the configuration of the wind field induced by the QSW1. This wave causes asymmetry along the edge of the polar vortex with formation of a region with a localized jet and high PV gradient. Smaller Rossby waves propagating along the vortex edge amplify then break as they enter the region where the mean wind and the PV gradient are weak. The role of the QSW1 is further confirmed by three-dimensional simulations. These simulations demonstrated that when the forcing due to QSW1 is present, wave breaking and the tongue develops in the region where the QSW1-induced winds are weak. If this forcing is suppressed, the polar vortex tends to be centered on the pole and the tongue of high PV does not develop.

This study was motivated by the observation of a balloon that was expelled from the vortex within a tongue of high PV during the VORCORE experiment. The mechanism responsible for the equator-ward transport of the balloon and its ejection into the surf zone, and the role of the QSW1 in creating the weak flow conditions that caused Rossby wave breaking near the vortex edge in a real atmosphere are presented for the first time. Finally, since the QSW1 dominates the stratospheric dynamics during the SH springtime, Rossby wave breaking similar to the event presented in this paper are expected to be more frequent and may significantly contribute to the climatological variability of tracers and air exchange between high and low latitudes during spring.

Acknowledgements. This work was partially supported by NSF grants ATM-0934592 and EF 1049251. We are thankful to the ECMWF for the free access to its archived analyses. We also thank the VORCORE team for the balloon data.

Topical Editor C. Jacobi thanks two anonymous referees for their help in evaluating this paper.

\section{References}

Agosta, E. A. and Canziani, P. O.: Austral Spring Stratospheric and Tropospheric Circulation Interannual Variability, J. Climate, 24, 2629-2647, doi:10.1175/2010JCLI3418.1, 2011.

Andrews, D. G., Holton, J. R., and Leovy, C. B.: Middle Atmosphere Dynamics, Edition 6, Academic Press, pp. 489, 1987.

Bowman, K. P.: Barotropic simulation of large-scale mixing in the Antarctic polar vortex, J. Atmos. Sci., 50, 2901-2914, 1993.

Dritschel, D. G.: Contour dynamics and contour surgery: numerical algorithms for extended, high-resolution modelling of vortex dynamics in two-dimensional, inviscid, incompressible flows, Computer Physics Reports, 10, 77-146, 1989.

Dritschel, D. G., Haynes, P. H., Juckes, M. N., and Shepherd, T. G.: The stability of a two-dimensional vorticity filament under uniform strain, J. Fluid Mech., 230, 647-665, 1991.

Esler, J. G. and Haynes, P. H.: Baroclinic Wave Breaking and the Internal Variability of the Tropospheric Circulation, J. Atmos. Sci., 56, 4014-4031, 1999.

Geller, M. A. and Wu, M. F.: Troposphere-stratosphere general circulation statistics, Transport Processes in the Middle Atmosphere, edited by: Visconti, G. and Garcia, R., Reidel, 3-17, 1987.

Hartman, D. L.: Stationary planetary waves in the Southern Hemisphere, J. Geophys. Res., 77, 437-471, 1977.

Haynes, P.: Stratospheric Dynamic, Annu. Rev. Fluid Mech., 37, 263-293, 2005. 
Haynes, P. H., Poet, D. A., and Shuckburgh, E. F.: Transport and mixing in kinematic and dynamically consistent flows, J. Atmos. Sci., 64, 3640-3651, 2007.

Hitchman, M. H. and Huesmann, A. S.: A seasonal climatology of Rossby wave breaking in the 320-2000-K layer, J. Atmos. Sci., 64, 1922-1940, 2007.

Karpetchko, A.,Kyrnd, E., and Knudsen, B. M.: Arctic and Antarctic polar vortices 1957-2002 as seen from the ERA-40 reanalyses, J. Geophys. Res., 110, D21109, doi:10.1029/2005JD006113, 2005.

Legras, B., Dritschel, D. G., and Calliol, P.: The erosion of a distributed two-dimensional vortex in a background straining flow, J. Fluid Mech., 441, 369-398, 2001.

Mariotti, A., Moustaoui, M., Legras, B., and Teitelbaum, H.: Comparison between vertical ozone soundings and reconstructed potential vorticity maps by contour advectionwith surgery, J. Geophys. Res., 102, 6131-6142, 1997.

McIntyre, M. E. and Palmer, T. N.: Breaking planetary waves in the stratosphere, Nature, 305, 593-600, 1983.

McIntyre, M. E. and Palmer, T. N.: The surf zone in the stratosphere, J. Atmos. Terr. Phys., 46, 825-849, 1984.

McIntyre, M. E. and Palmer, T. N.: A note on the general concept of wave breaking for Rossby and gravity waves, Pure Appl. Geophys., 123, 964-975, 1985.

Moustaoui, M., Teitelbaum, H., and Valero, F. P. J.: Ozone laminae inside the Antactic vortex produced by pole- ward filaments, Q. J. Roy. Meteorol. Soc., 129, 3121-3136, 2003a.

Moustaoui, M., Teitelbaum, H., and Valero, F. P. J.: Vertical displacements induced by quasi-stationnary waves in the southern Hemisphere during spring, Mon. Weather Rev., 131, 2279-2289, $2003 \mathrm{~b}$.

Nakamura, M. and Plumb, R. A.: The effects of flow asymmetry on the direction of Rossby wave breaking, J. Atmos. Sci., 51, 20312045, 1994.

Ndarana, T. and Waugh, D. W.: A Climatology of Rossby Wave Breaking on the Southern Hemisphere Tropopause, J. Atmos. Sci., 68, 798-811, 2011.

Orlanski, I.: Bifurcation in Eddy Life Cycles: Implications for storm track variability, J. Atmos. Sci., 60, 993-1023, 2003.

Peters, D. and Waugh, D. W.: Influence of barotropic shear on the poleward advection of upper tropospheric air, J. Atmos. Sci., 53, 3013-3031, 1996.

Peters, D. and Waugh, D. W.: Rossby Wave Breaking in the Southern Hemisphere Wintertime Upper Troposphere, Mon. Weather Rev., 131, 2623-2634, 2003.

Plumb, R. A.: Stratospheric transport, J. Meteorol. Soc. Jpn., 80, 793-809, 2002.

Plumb, R. A., Waugh, D. W., Atkinsons, R. J., Newman, P. A. Lait, L. R., Schoeberl, M. R., Browel, E. V., Simmons, A. J., and Lowenstein, M.: Intrusion into the lower stratospheric Arctic vortex during the winter of 1991/92, J. Geophys. Res., 99, 1089-1106, 1994.

Polvani, L. M. and Plumb, R. A.: Rossby wave breaking, filamentation and secondary vortex formation: The dynamics of a perturbed vortex, J. Atmos. Sci., 49, 462-476, 1992.
Randel, W. J.: The seasonal evolution of planetary waves in the Southern Hemisphere stratosphere and troposphere, Q. J. Roy. Meteorol. Soc., 114, 1385-1409, 1988.

Randel, W. and Newman, P. A.: The stratosphere in the Southern Hemisphere, in: Meteorology of the Southern Hemisphere, edited by: Karoly, D. J. and Vincent, D. G., Meteorol. Monogr., 27, 243-282, 1998.

Riviere, G. and Orlanski, I.: Characteristics of the Atlantic stormtrack eddy activity and its relation with the North Atlantic Oscillation, J. Atmos. Sci., 64, 241-266, 2007.

Shepherd, T. G.: Transport in the middle atmosphere, J. Meteorol. Soc. Jpn., 85B, 165-191, 2007.

Swanson, K. L.: Stationary wave accumulation and the generation of low-frequency variability on zonally varying flows, J. Atmos. Sci., 57, 2262-2280, 2000.

Swanson, K. L., Kushner, P. J., and Held, I. M.: Dynamics of barotropic storm tracks, J. Atmos. Sci., 54, 791-810, 1997.

Teitelbaum, H. and Moustaoui, M.: Observation of a tongue pulled out from the Antarctic vortex due to barotropic instability, SPARC 4th General Assembly, available at: http://www.atmosp.physics.utoronto.ca/SPARC/ SPARC2008GA/Posters/SessionB_P97_A68_Teitelbaum.pdf, 2008.

Teitelbaum, H., Moustaoui, M., Van Velthoven, P. F. J., and Kelder, H.: Decrease of total ozone at low latitudes in the southern hemisphere by a combination of linear and non-linear processes, Q. J. Roy. Meteorol. Soc., 124, 2625-2644, 1998.

Vincent, R. A., Hertzog, A., Boccara, G., and Vial, F.: Quasi-Lagrangian superpressure balloon measurements of gravity waves momentum fluxes in the polar stratosphere of both hemispheres, Geophys. Res. Lett., 34, L19804, doi:10.1029/2007GL031072, 2007.

Waugh, D. W. and Plumb, R. A.: Contour advection with surgery: A technique for investigating fine scale structure in tracer transport, J. Atmos. Sci., 51, 330-540, 1994.

Waugh, D. W. and Polvani, L. M.: Intrusions into the tropical upper troposphere, Geophys. Res. Lett., 27, 3857-3860, 2000.

Waugh, D. W. and Polvani, L. M.: Stratospheric polar vortices, in: The Stratosphere: Dynamics, Chemistry, and Transport, Geophys. Monogr. Ser., 190, edited by: Polvani, L. M., Sobel, A. H., and Waugh, D. W., pp. 43-57, AGU, Washington, D.C., 2010.

Waugh, D. W. and Randel, W. J.: Climatology of Arctic and Antarctic polar vortices using elliptical diagnostics, J. Atmos. Sci., 56, 1594-1613, 1999.

Waugh, D. W., Plumb, R. A., Atkinsons, R. J. , Schoeberl, M. R., Lait, L. R., Newman, P. A., Lowenstein, M., Toohey, D. W., Avallone, L. M., Webster, C. R., and May, R. D.: Transport out of the lower stratospheric Arctic vortex by Rossby wave breaking, J. Geophys. Res., 99, 1071-1088, 1994.

Wirth, V.: What causes the seasonal cycle of stationary waves in the southern stratosphere?, J. Atmos. Sci., 48, 1194-1200, 1991. 